\title{
Circular-to-planar transformations of high-perveance electron beams by asymmetric solenoid lenses
}

\author{
Stanley Humphries \\ Field Precision, PO Box 13595, Albuquerque, New Mexico 87192, USA \\ Steven Russell, Bruce Carlsten, and Lawrence Earley \\ Los Alamos National Laboratory, MS H851, Los Alamos, New Mexico 87545, USA \\ Patrick Ferguson \\ MDS Company, Oakland, California 94611, USA \\ (Received 4 March 2004; published 14 June 2004)
}

\begin{abstract}
The generation and transport of planar electron beams is a topic of increasing interest for applications to high-power, high-frequency microwave devices. This paper describes the use of solenoid lenses with elliptical pole apertures to transform a circular high-perveance beam to a planar configuration. The resulting transformation system has a short axial length and is easily fabricated. Furthermore, the approach allows use of a conventional electron gun. We designed lenses and simulated the dynamics of a beam system to drive a $95 \mathrm{GHz}$, traveling-wave tube experiment at Los Alamos National Laboratory. The three-dimensional self-consistent calculations indicate that a $0.5 \mu$ perv beam can be compressed to a height of less than $0.5 \mathrm{~mm}$ with sufficiently low emittance for transport through periodic-permanentmagnet or wiggler arrays.
\end{abstract}

DOI: 10.1103/PhysRevSTAB.7.060401

\section{INTRODUCTION}

We describe a simple method to transform a circular high-perveance electron beam to a planar configuration. Here the term planar implies that the height of the beam (vertical direction) is much smaller than the width (horizontal direction). Planar beams have potential application to the generation of high-power microwave radiation in devices such as sheet-beam klystrons and high-frequency traveling-wave tubes [1-11]. Flat beams have several advantages for the applications:

Higher net current can be transported for a given maximum focusing field.

The kinetic energy spread from space-charge effects is smaller.

The extended interaction area ensures smaller rf electric fields for a given power, reducing the possibility of breakdown.

Miniature rf structures (with scale size comparable to the beam height) can be used for high-frequency applications.

The last feature is critical for high-frequency traveling-wave tubes (TWT). The goal of the program $[12,13]$ at Los Alamos National Laboratory is the efficient generation of megawatts of microwave radiation in the frequency range of $95 \mathrm{GHz}$. This frequency corresponds to a vacuum wavelength of only $\lambda=3.2 \mathrm{~mm}$. An experiment under construction at the laboratory has the target parameters and requirements for beam transformation shown in Table I. In the listing (and in the remainder of the paper) we apply the convention that the planar beam propagates in $z$, has a large dimension in $x$, and a small
PACS numbers: $84.40 . \mathrm{Fe}$

dimension in $y$. The limit on angular divergence in the vertical direction is set by the properties of practical periodic-permanent-magnet or wiggler array transport systems. The constraints on the transport system are (i) the minimum distance between poles to accommodate the rf system, (ii) the limits of permanent-magnet materials, and (iii) the maximum cell length for stability. Horizontal focusing is not a major concern. Expansion forces are small because of the flat beam shape and image-charge effects in the nearby $\mathrm{rf}$ structure. Horizontal confinement can be accomplished easily with notched wiggler-magnet poles. We shall discuss studies of the transport system in a separate paper.

There are two possible approaches to the creation of thin planar beams: direct production by a sheet-beam gun with convergence in the vertical direction, and a conventional circular gun followed by a transformation element.

The sheet-beam approach presents several design challenges. The cathode and focusing electrode must have a carefully designed three-dimensional shape that compensates edge-focusing effects. Furthermore, threedimensional beam-generated forces act in the convergence section where the beam transitions to a flattened cross section. In contrast, the tools and procedures for the design of circular-beam guns are well known [14]. We show in this paper that the circular-to-elliptical conversion can be accomplished with a single optical element.

Quadrupole lens arrays are the common choice for transformations of low-perveance beams with elliptical cross sections [15]. It is challenging to employ quadrupole lenses for the high-current electron beams considered for high-power microwave generation [16]. The 
TABLE I. Parameters of the Los Alamos TWT experiment.

\begin{tabular}{lcc}
\hline \hline \multicolumn{1}{c}{ Quantity } & Symbol & Value \\
\hline Kinetic energy & $T_{e}$ & $120 \mathrm{keV}$ \\
Current & $I$ & $20 \mathrm{~A}$ \\
Generalized perveance & $K$ & $6.16 \times 10^{-3}$ \\
Radius of circular beam from gun & $r_{0}$ & $0.6 \mathrm{~cm}$ \\
Full height of planar beam & $2 y_{0}$ & $0.5 \mathrm{~mm}$ \\
Full width of planar beam & $2 x_{0}$ & $10.0 \mathrm{~mm}$ \\
Planar beam vertical angular divergence & $\Delta \theta$ & $0.06 \mathrm{rad}$ \\
\hline \hline
\end{tabular}

beams have a relatively large diameter and may expand significantly in a short axial distance. To illustrate, the length for a $50 \%$ expansion of a circular beam is [17]

$$
L_{z}=1.46814 \frac{r_{0}}{\sqrt{2 K}}
$$

where $r_{0}$ is the envelope radius at a waist and $K$ is the generalized perveance:

$$
K=\frac{e I}{2 \pi \varepsilon_{0} m_{e}(\gamma \beta c)^{3}} .
$$

The input circular beam in Table I would expand from a radius of 0.6 to $0.9 \mathrm{~cm}$ in a distance $L_{z}=7.94 \mathrm{~cm}$. The example illustrates the difficulty of controlling a highcurrent beam in an optical system with defocusing elements. Such a system requires large-aperture quadrupole lenses with a short axial length. The resulting nonlinear focusing forces may degrade the beam emittance.

As an alternative we describe the use of a conventional solenoid lens with elliptical pole-face apertures (Fig. 1). The shaped apertures generate unequal focusing forces in the horizontal and vertical directions. A solenoid lens has

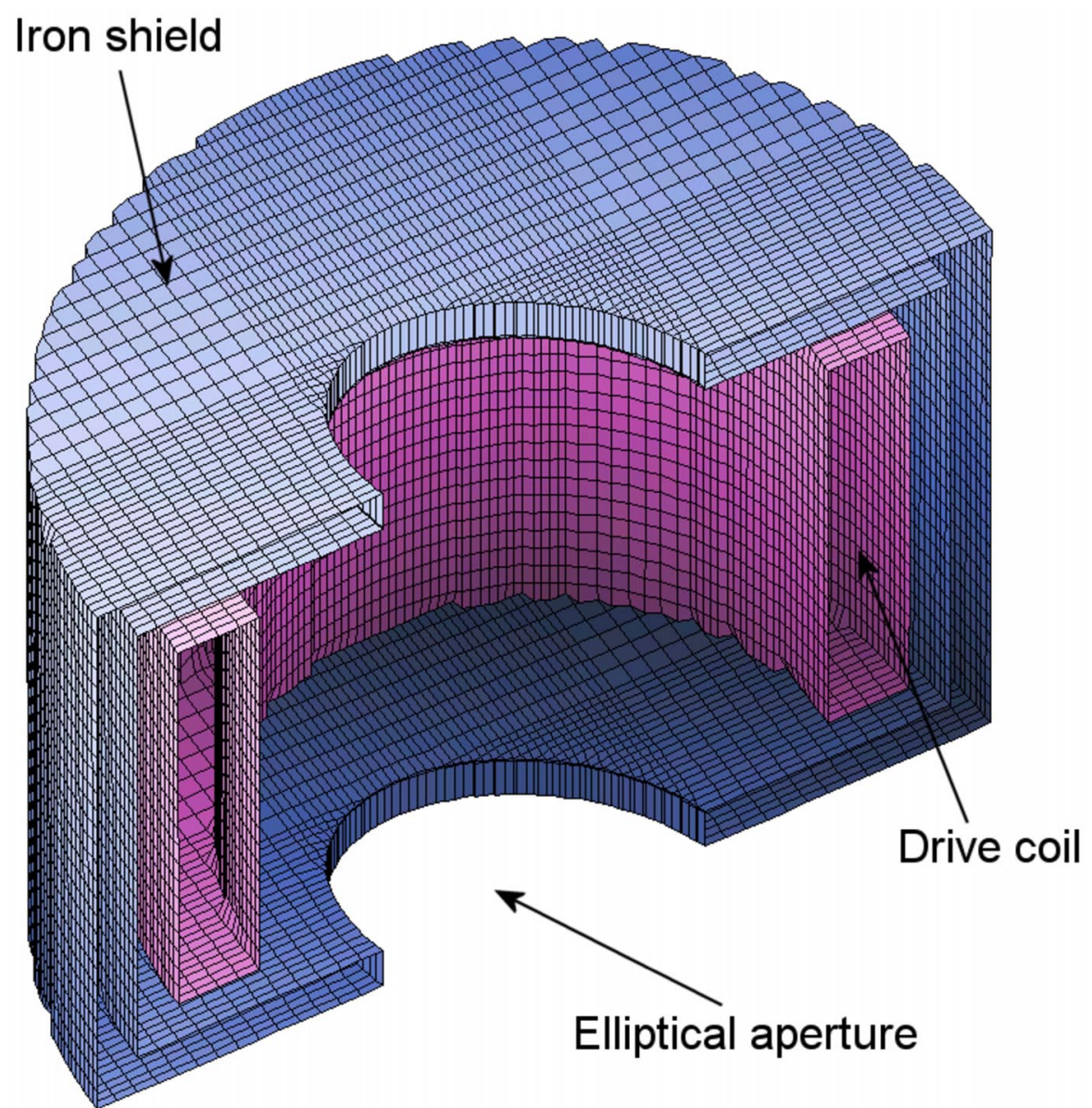

FIG. 1. (Color) Cutaway view of a solenoid lens with elliptical apertures showing the conformal mesh structure for the finiteelement field calculation. The elliptical axes are rotated so that the exit beam is parallel to the $x-y$ axes of the simulation. 
the advantage that it provides continuous focusing in both directions. Therefore, the beam transformation can be accomplished in a short distance. There are two main feasibility issues:

Can the necessary force asymmetry be generated in a practical device?

Are transverse forces sufficiently linear to meet emittance requirements?

Section II covers some theoretical background to guide the design of the lens. The Kapchinskij-Valdimirskij (KV) equations [18] are used to study the propagation of circular high-perveance beams that have passed through a thin lens with unequal focal lengths $f_{x}$ and $f_{y}$ in the horizontal and vertical directions. The calculations show that if $f_{y}<f_{x}$ and if $f_{y}$ is sufficiently small, a high-current circular beam can be focused to a line of zero thickness in $y$. Furthermore, it is possible to achieve the desired beam width in $x$ at the vertical focal point by proper choice of the lens strength and the ratio $f_{x} / f_{y}$. Although it is not possible to achieve simultaneous waist points in $y$ and $x$, the horizontal envelope angle is small and should have little effect on downstream transport. Section III discusses the lens design. A device suitable for the experimental parameters of Table I is compact and easy to fabricate. Single-particle orbit calculations are used to determine the relationship between the aperture geometry and the focal length ratio. The calculations also give information on focal limits set by nonlinear forces. Section IV summarizes the main production simulations that include effects of self-consistent three-dimensional beam-generated forces. The calculations indicate that a practical, single-component optical system can accomplish the beam transformation of Table I.

\section{ELLIPTICAL BEAM TRANSFORMATIONS WITH ANISOTROPIC LENSES}

The KV equations describe the propagation of a paraxial, uniform current-density elliptical beam in free space. The equations have the following form for a beam with zero emittance moving through a drift region with no focusing forces [19]:

$$
\begin{aligned}
& \frac{d^{2} X}{d z^{2}}=\frac{2 K}{X+Y}, \\
& \frac{d^{2} Y}{d z^{2}}=\frac{2 K}{X+Y} .
\end{aligned}
$$

The quantity $X$ is the horizontal envelope half-width, $Y$ is the vertical half-width, and $K$ is the generalized perveance of Eq. (2). Equations (3) and (4) also apply exactly within a metal vacuum chamber if the chamber cross section follows a contour line of the space-charge potential. The equations give a good approximation inside a circular vacuum chamber that has a radius large compared to $X$ and $Y$.
Equations (3) and (4) imply that a beam with uniform current density always maintains an elliptical cross section. A second implication is that it is impossible to focus a circular beam to an elliptical waist $\left(X^{\prime}=d X / d z=0\right.$, $Y^{\prime}=d Y / d z=0$ ) using a thin lens with different focal strengths in $x$ and $y$. Consider a beam expanding upstream in free space from an ideal waist where $X \neq Y$. Equations (3) and (4) imply that the changes in the envelope widths are equal. Because the initial values differ, the beam cross section can never be circular $(X=Y)$. Therefore, the practical goal in the following studies is to ensure that the beam has the correct width $X$ at the waist in $y$ and a small value of envelope angle $X^{\prime}$.

The KVequations can yield insight into the effect of an elliptical solenoid lens on a high-perveance beam. Approximating the solenoid as a thin lens with unequal focal lengths $f_{x}$ in $x$ and $f_{y}$ in $y$, Eqs. (3) and (4) can be solved numerically with the initial conditions $X_{0}=Y_{0}=$ $r_{0}$ and

$$
\begin{aligned}
& X_{0}^{\prime}=-\frac{r_{0}}{f_{x}}, \\
& Y_{0}^{\prime}=-\frac{r_{0}}{f_{y}} .
\end{aligned}
$$

We performed calculations using the parameters from Table I with one exception - the initial radius was taken as $r_{0}=0.8 \mathrm{~cm}$ to approximate the effect of beam expansion between the gun output and the lens center. The calculations covered a range of $Y_{0}^{\prime}$ for a given ratio of focal lengths. Figures 2 and 3 show results for $f_{x} / f_{y}=$ $5 / 3$. Figure 2 shows $Y$ at the point of minimum vertical thickness and the axial distance from the lens versus the initial angle $Y_{0}^{\prime}$ (proportional to the vertical lens strength). Figure 3(a) shows values of the envelope angles $X^{\prime}$ and $Y^{\prime}$ at the point of minimum vertical thickness while Fig. 3(b) plots the horizontal envelope width $X$. At low lens strength the beam behavior was similar to that of a circular beam. It compressed to a vertical waist point where $Y>0$ and $Y^{\prime}=0$. Above a critical lens strength, the beam focus changed to a mode where the minimum thickness dropped to zero with $Y^{\prime}>0$ at the focus. We can understand the transition by comparing the focal properties of circular and flat high-current beams. A focused circular beam compresses in both $x$ and $y$. The repulsive force associated with the increasing space-charge density counteracts the inward inertia so that the beam reaches a waist. A flat beam compresses only in $y$-the repulsive force on the envelope does not increase. As a result it is possible to focus a planar beam to a line of zero thickness. The solutions show that an elliptical beam acts like a planar beam at high aspect ratio.

Figures 2(a) and 3(a) show that both the thickness $Y$ and envelope angle $Y^{\prime}$ approached zero at a critical lens strength corresponding to $Y_{0}^{\prime}=-0.145 \mathrm{rad}$ (for the parameters of Table I). In this case the distance from the lens 

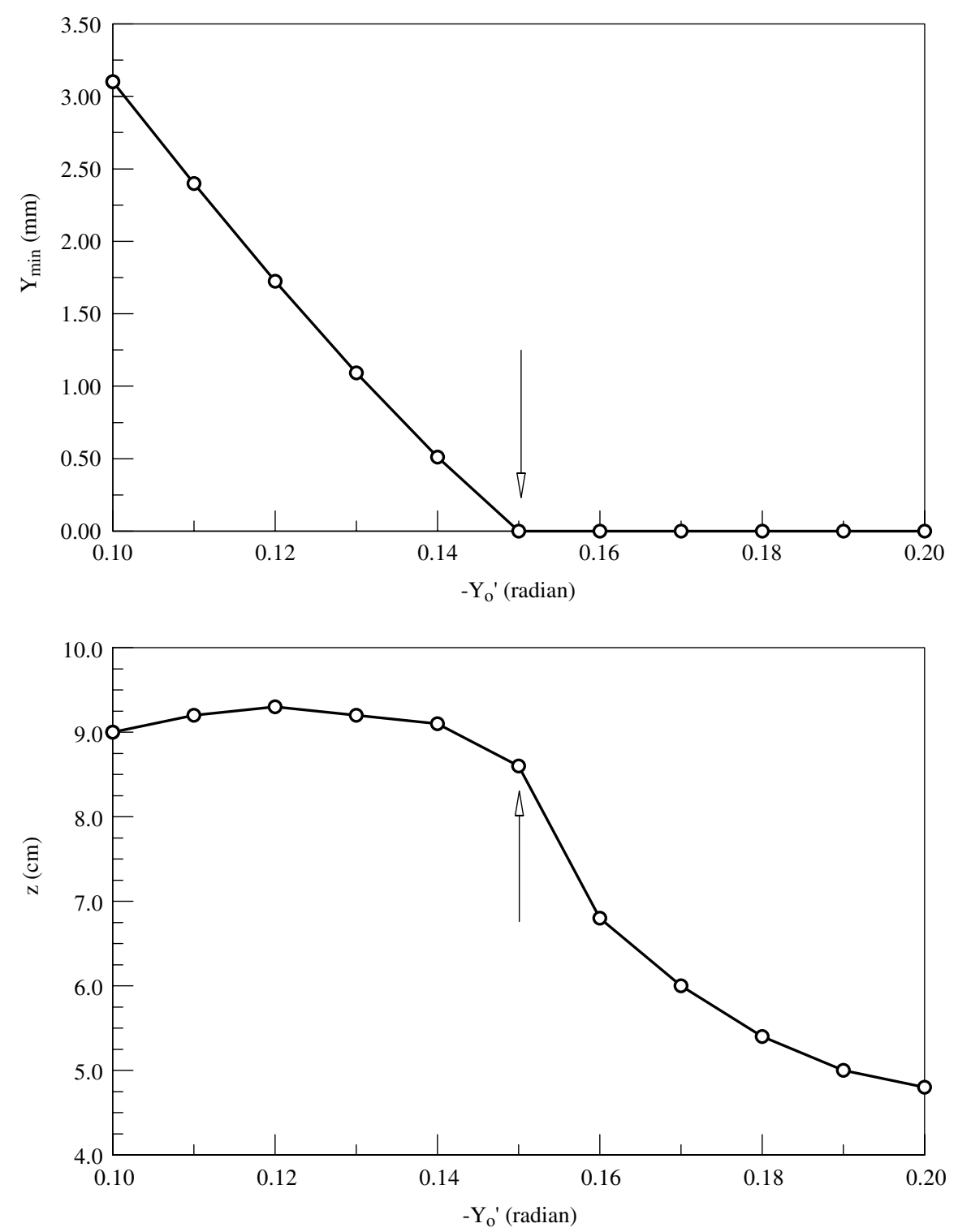

FIG. 2. Transformation by an anisotropic thin lens, solutions of the KV equations. Variations with initial vertical envelope angle $Y_{0}^{\prime}$ for $f_{x} / f_{y}=5 / 3$. Arrows show the optimal focus condition. (a) Minimum vertical half-width $Y_{\min }$. (b) Axial distance from the lens to the vertical focus.

to the focal point was $8.85 \mathrm{~cm}$. This state is best for matching into a downstream transport system. We shall refer to the condition as the optimal focus. The figures show that the minimum vertical thickness increased at a lower lens strength, while the envelope angle at minimum thickness increased at a higher lens strength. In the latter case the inward inertia dominated and the beam reached a ballistic focus where orbits crossed the axis. Table II shows scaling of parameters at the optimal focus with the focal length ratio $f_{x} / f_{y}$.

The results of Figs. 2 and 3 and Table II suggest a procedure to design an anisotropic solenoid lens:

Choose a value of $f_{x} / f_{y}$ to give the desired horizontal width at the optimal focal point.
Design a lens with the desired focal length ratio. The lens must be short enough to provide sufficient distance for isolation from the magnetic fields of the transport system. Set the lens strength to give the required value of $Y_{0}^{\prime}$ for the optimal focus.

Determine the spread in vertical angles at the optimal focus caused by spherical aberration.

Design a periodic planar-beam transport system with the entrance point at the optimal focus. The vertical focusing force of the transport system must be sufficient to maintain the beam height in the presence of spacecharge forces and angular divergence. Horizontal focusing forces must be strong enough to compensate the initial envelope divergence. 

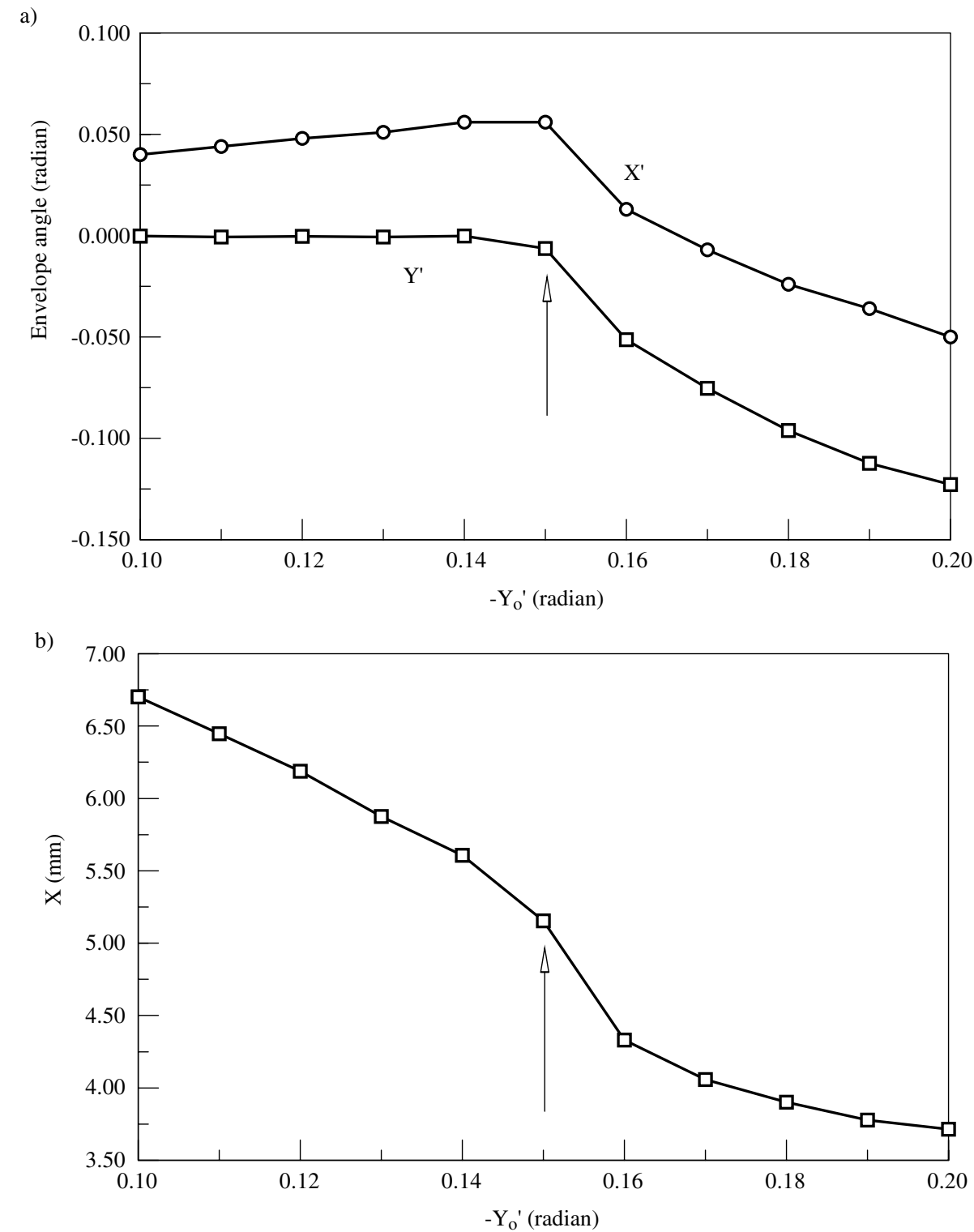

FIG. 3. Transformation by an anisotropic thin lens, solutions of the KV equations. Variations with initial vertical envelope angle $Y_{0}^{\prime}$ for $f_{x} / f_{y}=5 / 3$. Arrows show the optimal focus condition. (a) Envelope angles $X^{\prime}$ and $Y^{\prime}$ at the point of minimum vertical thickness. (b) Horizontal half-width $X$ at the point of minimum vertical thickness.

TABLE II. Properties of the optimal focus as a function of lens anisotropy.

\begin{tabular}{ccccc}
\hline \hline$f_{x} / f_{y}$ & $Y_{0}^{\prime}(\mathrm{rad})$ & $z\left(Y_{\min }\right)(\mathrm{cm})$ & $X\left(Y_{\min }\right)(\mathrm{mm})$ & $X^{\prime}\left(Y_{\min }\right)(\mathrm{rad})$ \\
\hline 1.250 & -0.170 & 6.90 & 2.35 & 0.029 \\
1.667 & -0.150 & 8.60 & 5.16 & 0.056 \\
2.500 & -0.140 & 9.00 & 7.55 & 0.062 \\
\hline \hline
\end{tabular}

\section{FOCAL PROPERTIES OF AN ELLIPTICAL SOLENOID LENS}

We designed a benchmark solenoid lens using the three-dimensional finite-element MAGNUM code [20].
We then used the OMNITRAK code [20] to trace orbits to characterize the lens. The lens geometry is shown in Fig. 1. The field was created by a set of circular coils with average radius $4.5 \mathrm{~cm}$. The coils were located inside a soft iron shield with cylindrical symmetry. The shield consisted of a support cylinder (inner radius $5.0 \mathrm{~cm}$, thickness $0.5 \mathrm{~cm}$ ) and two end pieces with apertures $(0.5 \mathrm{~cm}$ thick). The lens had a total length of $6.0 \mathrm{~cm}$. With circular apertures the assembly acted as a conventional solenoid lens. Elliptical apertures gave different focal properties in the $x$ and $y$ directions. The design goals were simplicity of fabrication and maximum versatility. We found that the required range of focal anisotropy could be achieved with elliptical apertures of a 
TABLE III. Focal properties as a function of aperture aspect ratio.

\begin{tabular}{lccccc}
\hline \hline$R_{x}(\mathrm{~cm})$ & $R_{y}(\mathrm{~cm})$ & $R_{x} / R_{y}$ & $f_{x}(\mathrm{~cm})$ & $f_{y}(\mathrm{~cm})$ & $f_{x} / f_{y}$ \\
\hline 2.000 & 2.000 & 1.000 & 10.3 & 10.3 & 1.000 \\
2.100 & 1.905 & 1.102 & 12.3 & 9.0 & 1.367 \\
2.200 & 1.818 & 1.210 & 14.2 & 8.1 & 1.753 \\
2.300 & 1.739 & 1.323 & 16.6 & 7.4 & 2.240 \\
\hline \hline
\end{tabular}

modest aspect ratio. In comparing focal properties, the area of the aperture was maintained at a constant value equal to that of a circular aperture of radius of $2.0 \mathrm{~cm}$. The relatively small aperture size ensured localization of the lens fringing fields to minimize interference with the upstream electron gun and downstream planar transport system. The limit on the aspect ratio of elliptical apertures was set by the size of the beam transport vacuum tube (taken as a thin cylinder with $1.5 \mathrm{~cm}$ radius).

Because of beam rotation it was necessary to include all quadrants in $x-y$ in the magnetic field calculation. The resulting mesh contained about 432000 nodes. The element size in the beam propagation region was $0.10 \mathrm{~cm}$ in $x$ and $y$ and $0.25 \mathrm{~cm}$ in $z$. The run time for each field calculation was about $7 \mathrm{~min}$ on a $2 \mathrm{Ghz}$ computer. The goal of the first series of orbit calculations was to determine the relationship between the aperture geometry and the relative horizontal and vertical focal lengths. We used a small input beam radius $(0.25 \mathrm{~cm})$ to differentiate the paraxial focal lengths from the effects of spherical aberration. A drive current of $1750 \mathrm{~A}$-turns gave a point focus approximately $4.0 \mathrm{~cm}$ from the lens exit for a circular aperture. Table III lists focal lengths in the $x$ and $y$ directions for some choices of the aperture shape. The

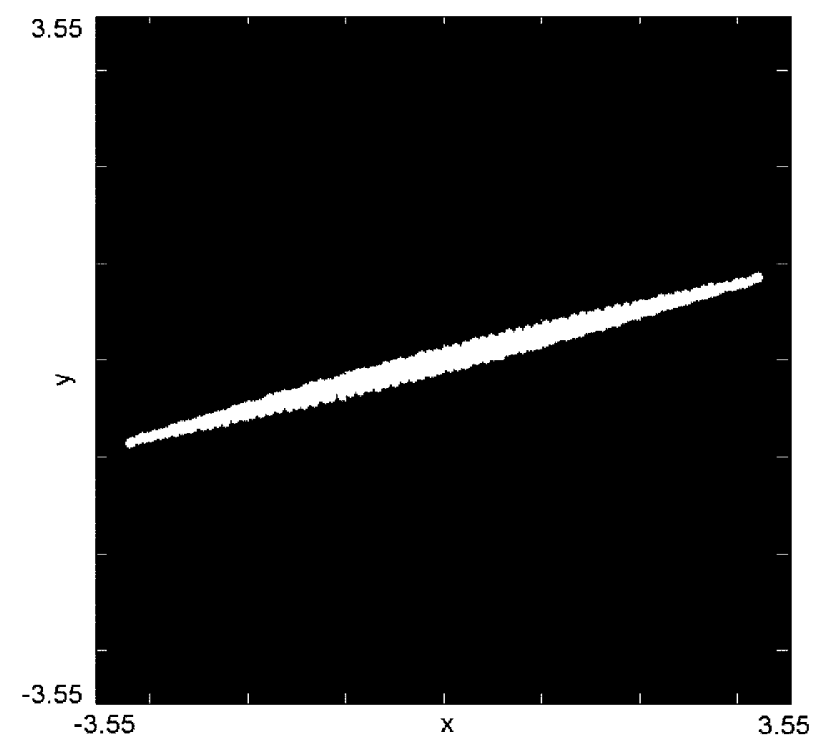

FIG. 5. Beam distribution generated by OMNITRAK at the vertical focal point. Initial beam radius: $0.8 \mathrm{~cm} . R_{x}=$ 2.200, $R_{y}=1.818$. Dimensions in mm. Fiducial intervals in $x$ and $y: 1.0 \mathrm{~mm}$.

thin-lens focal lengths were taken as the distance from the lens midplane to the focal points. Note that the $x$ and $y$ directions in the exit space were rotated approximately $15^{\circ}$ with respect to the entrance coordinates. In all cases the axes of the elliptical apertures in both the entrance and exit plates were parallel to the entrance coordinates. Figure 4 shows $f_{x} / f_{y}$ as a function of $R_{x} / R_{y}$ for the baseline lens design. The variation is approximately linear over the allowed range. The choice $R_{x}=$ $2.200 \mathrm{~cm}$ and $R_{y}=1.818 \mathrm{~cm}$ gave a value of $f_{x} / f_{y}$ close

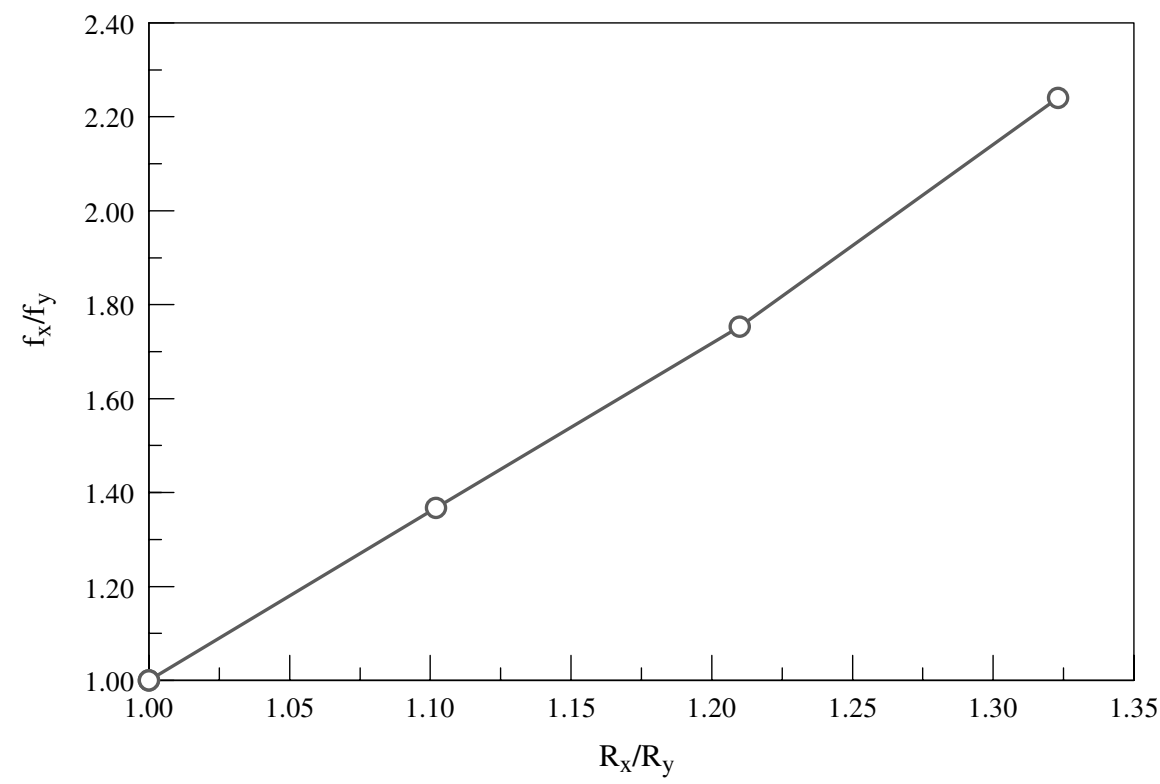

FIG. 4. Ratio of focal lengths in the horizontal and vertical directions as a function of the aspect ratio of the elliptical apertures for the baseline lens design. 
to the figure of 1.667 in Table II corresponding to a horizontal full width of approximately $1.0 \mathrm{~cm}$ at the point of optimal vertical focus. This geometry was used as a starting point for the full beam computations described in the following section.

A second issue addressed with single-particle calculations was the limitation on vertical focusing set by spherical aberration. We performed a simulation with $f_{x} / f_{y}=1.753$ and an input beam radius of $0.8 \mathrm{~cm}$ at a lens current of 1750 A-turns. The minimum vertical envelope width occurred at a distance of $4.9 \mathrm{~cm}$ from the lens exit, slightly upstream from the paraxial focal point of $5.1 \mathrm{~cm}$. Figure 5 shows the beam distribution in a plane normal to $z$ at the vertical focal point. The full width of the beam is $\sim 0.2 \mathrm{~mm}$, within the required width of $0.5 \mathrm{~mm}$. The implication is that aberrations in the elliptical lens with $2.0 \mathrm{~cm}$ radius are sufficiently small to focus a beam with $0.8 \mathrm{~cm}$ entrance radius.

\section{SIMULATIONS OF HIGH-PERVEANCE BEAM TRANSFORMATION BY ELLIPTICAL SOLENOID LENSES}

The full features of the OMNITRAK code were used to simulate the transformation properties of the elliptical lenses described in Sec. III for the high-current beam of Table I. The input beam parameters were confirmed by simulations of the electron gun using the twodimensional TRAK [20] code. The results showed that we could generate a beam at the exit of the gun with a waist of radius $0.6 \mathrm{~cm}$, uniform current density, and negligible emittance. We picked a relatively short distance of $4.0 \mathrm{~cm}$ from the gun exit to the lens entrance to minimize beam expansion and to avoid spherical aberration effects in the lens. The goal of the simulations was to compress the beam to a line focus with the desired dimensions at a point at least $4.0 \mathrm{~cm}$ from the lens exit. In the following discussion, the location $z=0.0$ corresponds to the lens midplane. The variable parameters were (i) the lens current and (ii) the aspect ratio of the aperture ellipses. The ellipse area had the fixed value $\pi(2.0)^{2} \mathrm{~cm}^{2}$. Beam space-charge fields were computed on an electric-field mesh that represented a grounded vacuum chamber of radius $1.5 \mathrm{~cm}$ extending from $z=$ $-7.0 \mathrm{~cm}$ ( $4.0 \mathrm{~cm}$ upstream from the lens entrance) to $z=$ $12.0 \mathrm{~cm}$. Neumann boundary conditions were applied at the entrance and exit planes of the electric-field solution volume. The mesh had approximately 250000 nodes. The OMNITRAK simulation used 1163 model particles with equal current weighting to represent the circular beam. A run required 20 cycles of orbit tracing and field recalculation for convergence. Although the code has the capability to represent complete three-dimensional variations of beam-generated magnetic fields, we used the simpler relativistic mode to reduce run time. In this mode the code performs only the electric-field calculation and scales forces transverse to the $z$ axis by a factor $1 / \gamma^{2}$ to include the effect of beam-generated magnetic field. The quantity $\gamma$ is the relativistic energy factor of beam

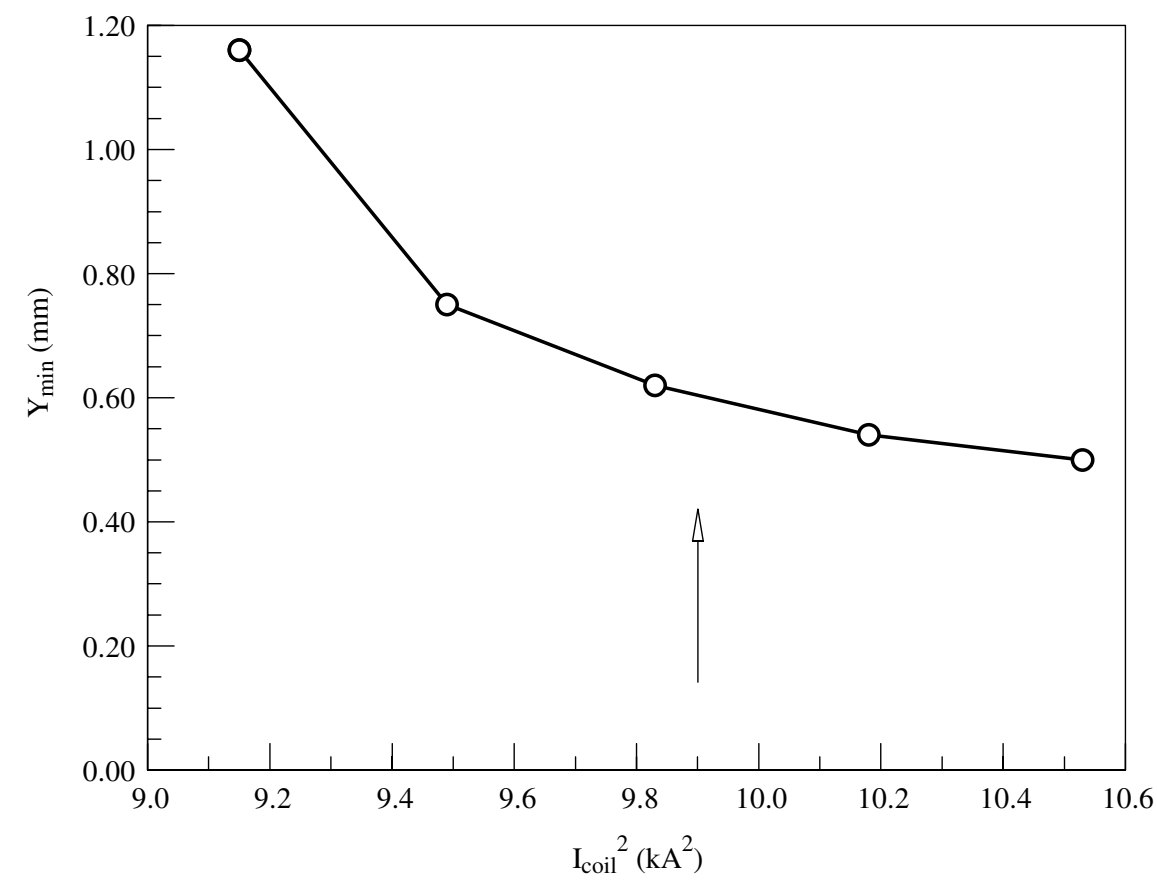

FIG. 6. Minimum vertical half-width as a function of the relative lens strength for $R_{x}=2.200, R_{y}=1.818$. Arrow shows the approximation optimal focal point. 

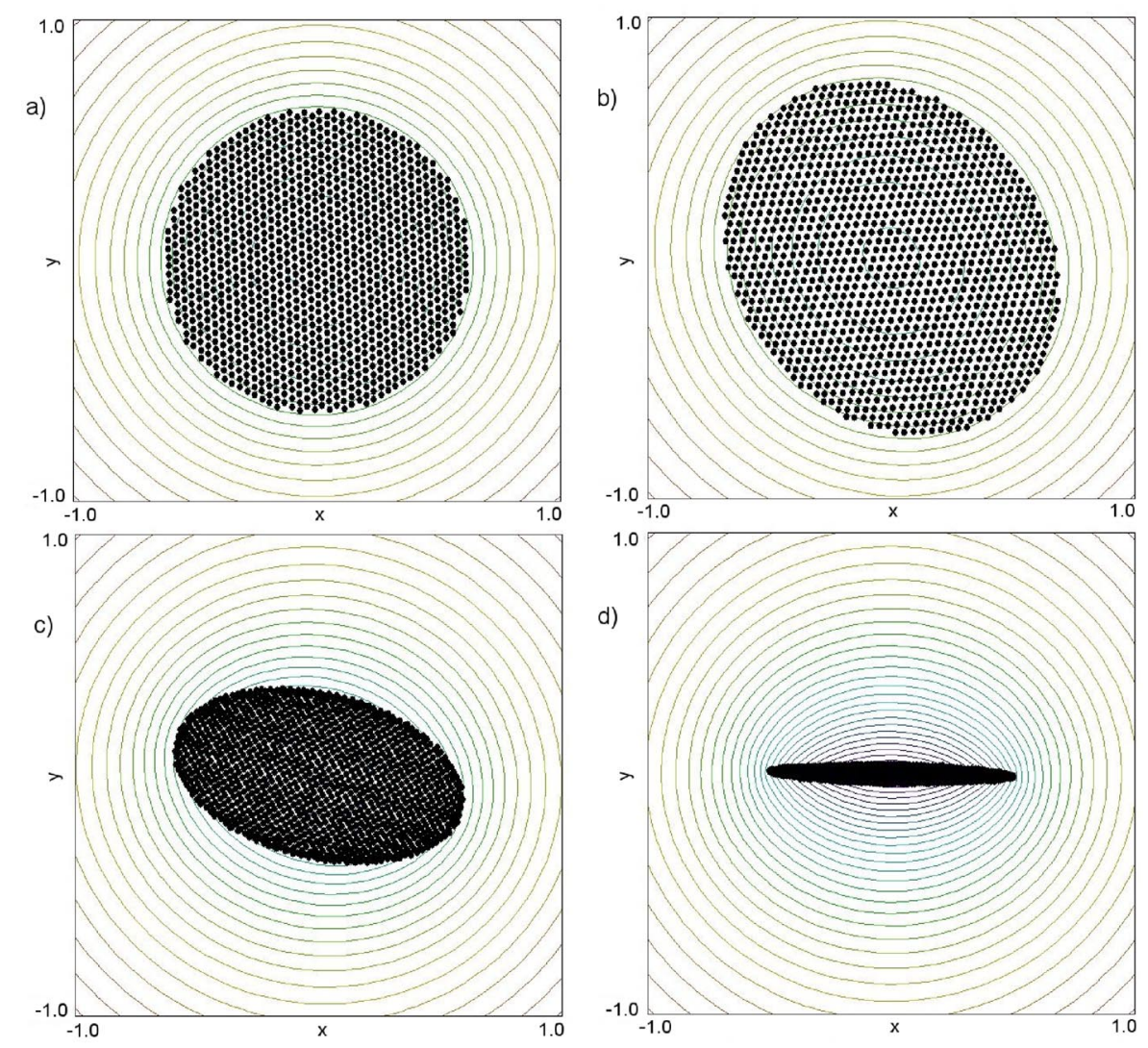

FIG. 7. (Color) Simulation particle distributions projected in planes normal to $z$ and contours of the beam-generated electrostatic potential. (a) Gun output at the entrance to the transformation system, $z=-7.0 \mathrm{~cm}$. (b) Lens midplane, $z=0.0 \mathrm{~cm}$. (c) Lens exit, $z=3.0 \mathrm{~cm}$. (d) Vertical focal point, $z=7.6 \mathrm{~cm}$.

particles. The mode is valid in transport simulations when (i) motion is paraxial, (ii) there is a small spread in axial energy, and (iii) the electric and magnetic fields arise solely from the presence of the beam. A complete run required $20 \mathrm{~min}$ on a $2 \mathrm{GHz}$ computer.

Following the results of Sec. III, we initially used an aperture with $R_{x}=2.200 \mathrm{~cm}$ and $R_{y}=1.818 \mathrm{~cm}$. Several simulations were performed over a range of values for the lens current $I_{\text {coil }}$. Values of the vertical thickness at the focal point were plotted to identify the value of $I_{\text {coil }}$ corresponding to the optimal focus. Figure 6 shows calculated values of $Y_{\min }$ at the vertical focal point over a range of coil current from 2750 to 2950 A-turns. For comparison to the plots in Figs. 2 and 3, the quantity $-I_{\text {coil }}^{2}$ (proportional to the lens focusing strength) is plotted on the abscissa. Compared to the single-particle simulations of Sec. III, a higher lens current was required to counteract the space-charge forces. The beam rotation at the highest value of $I_{\text {coil }}$ was $43^{\circ}$. Inspection of Fig. 6 shows that the transition to a space-charge-limited vertical focus occurred when the magnitude of the coil current dropped below about $2850 \mathrm{~A}\left(I_{\text {coil }}^{2}=8.12 \mathrm{kA}^{2}\right)$. Note that the transition was not sharp and that the beam thickness did not fall to zero at higher current. The minimum thickness of about $0.5 \mathrm{~mm}$ represents the combined effects of lens aberrations and numerical noise in the calculation of beam-generated forces within the thin beam. The horizontal full width at $I_{\text {coil }}=2850$ A-turns was $9.1 \mathrm{~mm}$, close to the target value of $10.0 \mathrm{~mm}$. This drive current corresponded to a peak lens field of $B_{z}=$ $0.0618 \mathrm{~T}$.

We modified the solution to conform more closely with the target parameters of Table I. Following the scaling in Table III, the aperture aspect ratio was increased $\left(R_{x}=\right.$ $\left.2.225, R_{y}=1.798\right)$ to shift the optimum focal point downstream and to broaden the beam in the horizontal direction. A search similar to that shown in Fig. 6 gave a drive current for optimal focus of about $I_{\text {coil }} \cong 2800 \mathrm{~A}$ turns. At this value the beam rotation between the entrance and exit planes was $40^{\circ}$. To facilitate analysis of the output beam, we rotated the lens apertures $-40^{\circ}$ to align the final beam ellipse with the simulation coordinate axes (Fig. 1). Figure 7 shows a sequence of $x-y$ beam distributions at different axial locations through the transformation. The optimal vertical focal point was about $7.6 \mathrm{~cm}$ from the lens midplane $(4.6 \mathrm{~cm}$ from the lens exit). At this location the horizontal full width was close to $1.0 \mathrm{~cm}$ and the vertical full width was $0.68 \mathrm{~mm}$.

Figure 8 shows phase-space distributions in $x$ and $y$ near the point of optimal vertical focus. The distortion of 

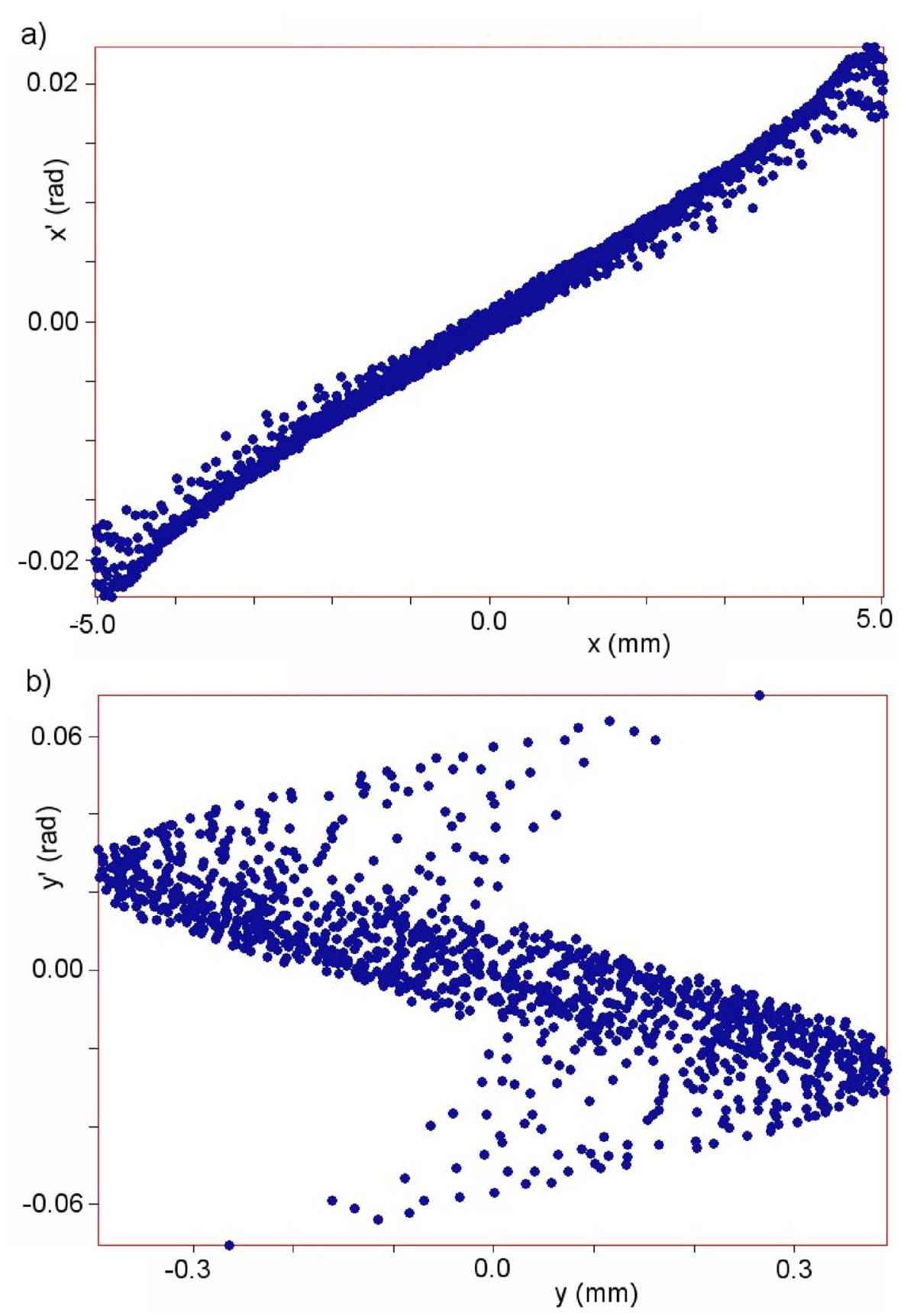

FIG. 8. (Color) Phase space distributions at the optimal focus. (a) Horizontal, $x-x^{\prime}$. (b) Vertical, $y-y^{\prime}$.

the $y-y^{\prime}$ distribution arises from spherical aberration. Despite the lens imperfections, $99 \%$ of the beam current was contained within an angular spread of $\pm 0.05 \mathrm{rad}$. An analysis of the beam distribution at the optimum focus gives the following root-mean-squared quantities: displacement in $x: 2.713 \mathrm{~mm}$; displacement in $y: 0.218$ $\mathrm{mm}$; angular spread in $x$ : $0.0113 \mathrm{rad}$; angular spread in $y$ : $0.0216 \mathrm{rad}$; emittance in $x: 1.1621 \times 10^{-5} \pi \mathrm{m} \mathrm{rad}$; emittance in $y: 1.3326 \times 10^{-5} \pi \mathrm{m} \mathrm{rad}$. The nonzero values of exit emittance represent the cumulative effect of lens aberrations, code noise, and nonlinear space-charge forces.
In conclusion, solenoid lenses with elliptical apertures may be effective for transforming high-perveance circular electron beams into flat elliptical beams. It appears feasible to create beams with a small vertical width and an angular divergence consistent with transport in the Los Alamos traveling-wave tube experiment. The solenoid lens discussed in this report is easy to fabricate - the coils and housing have cylindrical symmetry. Anisotropic focusing forces result solely from the elliptical apertures in the removable end flanges. The solenoid lens transformation system is compact-the length from the electron gun to the 
entrance of the planar-beam transport system is only $14.6 \mathrm{~cm}$.

[1] J. Joe, S. F. Chang, J. E. Scharer, and J. H. Booske, Microwave Opt. Tech. Lett. 4, 443 (1991).

[2] J. H. Booske and M. A. Basten, IEEE Trans. Plasma Sci. 27, 134 (1999).

[3] B. D. McVey, M. A. Basten, and J.H. Booske, IEEE Trans. Microwave Theory Tech. 42, 995 (1994).

[4] J. Joe, L. J. Louis, J. E. Scharer, J. H. Booske, and M. A. Basten, Phys. Plasmas 4, 2707 (1997).

[5] E. R. Colby, G. Caryotakis, W. R. Fowkes, and D. N. Smithe, in High-Energy Density Microwaves, edited by R. M. Phillips, AIP Conf. Proc. No. 474 (AIP, New York, 1998), p. 74.

[6] G. Caryotakis, in Proceedings of the 5th Workshop on High Energy Density and High-Power RF, edited by Bruce Carlsten, AIP Conf. Proc. No. 625 (AIP, New York, 2001), p. 1.

[7] P. B. Wilson, in Proceedings of the International Workshop on RF Pulsed Sources for Linear Colliders (Stanford Linear Accelerator Center, Palo Alto, 1996).

[8] M.E. Read et al., in Proceedings of the Particle Accelerator Conference, Portland, OR, 2003 (IEEE, Piscataway, NJ, 2003).

[9] B. E. Carlsten, Phys. Plasmas 8, 4585 (2001); 9, 1790 (2002); 9, 5088 (2002).

[10] M. A. Basten, J. H. Booske, and J. Anderson, IEEE Trans. Plasma Sci. 22, 960 (1994).

[11] M. A. Basten and J. H. Booske, J. Appl. Phys. 85, 6313 (1999).

[12] B. E. Carlsten, L. M. Earley, W. B. Haynes, and R. M. Wheat, in Proceedings of the 5th Workshop on High Energy Density and High-Power RF (Ref. [6]), p. 117.

[13] B. E. Carlsten,S. J. Russell, L. M. Earley, J. M. Potter, P. Ferguson, and S. Humphries, Jr., in Proceedings of the 6th Workshop on High Energy Density and HighPower RF, Berkeley Springs, edited by S. Gold and G. Nusinovich, AIP Conf. Proc. No. 691 (AIP, New York, 2003), p. 349.

[14] See, for instance, J. R. Pierce, Theory and Design of Electron Beams (Van Nostrand, Princeton, 1954); J. F. Gittins, Power Traveling-Wave Tubes (Elsevier, Amsterdam, 1964); A.S. Gilmour, Jr., Principles of Traveling-Wave Tubes (Artech House, Norwood, 1994); R. True, Electron Beam Formation, Focusing and Collection in Microwave Tubes, Handbook of Microwave Technology Vol. 1 (Academic Press, New York, 1995), p. 497.

[15] See, for instance, R. Servranckx and K. L. Brown, in Circular Machine Design Techniques and Tools, Physics of Particle Accelerators, edited by M. Month and M. Dienes, AIP Conf. Proc. No. 153 (2 volumes) (AIP, New York, 1987).

[16] M. A. Basten, J. H. Booske, J. Anderson, and J. E. Scharer, Proc. Soc. Photo-optical Inst. Engr. 2557, 262 (1995).

[17] See, for instance, J. D. Lawson, The Physics of ChargedParticle Beams (Clarendon Press, Oxford, 1988), 2nd ed., Sect. 3.2.8.

[18] I. M. Kapchinskii, Dynamics in Linear Resonance Accelerators (Atomizdat, Moscow, 1966).

[19] See, for instance, S. Humphries, Charged Particle Beams (Wiley Interscience, New York, 1990), Sect. 9.3.

[20] MAGNUM, OMNITRAK, and TRAK software, copyright Field Precision, Albuquerque, NM. 